\title{
Electronic Health Record Management: Expectations, Issues, and Challenges
}

\author{
Mathai $\mathbf{N}^{*}$, Shiratudin MF and Sohel F
}

Department of Information Technology, Murdoch University, Perth, Australia

\begin{abstract}
Health record management is an important and challenging task. Utilization of technologies in health care, particularly the use of Electronic Health Records (EHR) offers a wide variety of benefits. Better healthcare is provided by EHR by improving all aspects of health care. This paper aims to do an extensive review of existing literature on the issues and expectations concerning consumers and physicians in the development of EHR. In this paper, based on the issues, recommendations are made, and future directions explored.
\end{abstract}

Keywords: Electronic Health Records (EHR); Digital health records; Health care

\section{Introduction}

Electronic Health Records (EHR) consists of a repository of information concerning the health status of individuals. In an EHR, health records are created and managed in digital formats [1]. An EHR of a patient can contain medical history including operations, hospitalizations, medications, past diagnostic follow-up, laboratory results, radiology reports, and relevant health care information $[2,3]$. It is a secure and reliable source of clinical information where health records can be safely shared.

The history of clinical documentation is based on paper based records, and they are cumbersome and ineffective [2]. Efficient data retrieval is possible from EHR systems in ways that paper documentation is unable to do [1]. Traditional medical records have restrictions in allowing a global vision of the patient's health conditions. An EHR instead, aims to gather health data, potentially generated by different sources at different times, and share those data with relevant healthcare systems [4]. The sharing of healthcare information between providers using EHR has led to improved outcomes of care and reduced clinical errors [1].

The success of a health care system depends on maintaining patent's trust. When patients lose their trust in the privacy of their health information kept by the providers, it leads to potentially dangerous steps including patients not properly disclosing important health information, and can even culminate in consumer's refusal to the EHR system [2].

Properly designed EHR systems eliminate the need to take down transcriptions on paper and can well organize physician workflows resulting in increased efficiency and productivity. The use of EHR systems has helped the healthcare providers with the better exchange of laboratory results, scans reports and improved communication method with patients [3]. A well-implemented health care system will reduce the inefficiencies, and promote a better health care. EHR standards are one of the essential building blocks for health care reform and an important component that supports efficient exchange of information between providers and health care organizations $[5,6]$.

Efficient healthcare delivery is about providing care without the wasteful use of resources. Duplicate testing and prescribing can be reduced by the EHR's ability to transfer patient information automatically between health care sites. Automatic reminders will reduce errors, improve productivity, and benefit patient care [7].
The ability of EHR to share information electronically provides a boost in quality in healthcare management. The main goals of EHR include providing a secure, reliable, and efficient way to register, gather and process all the clinical data related to the patient. Also, it supports the actions related to the clinical practice and patient treatment [8]. When optimally implemented, EHR holds a tremendous potential benefit for healthcare systems, and can enhance how patient data are documented and organized. It is therefore important to study and find out the current issues regarding EHR to make it more efficient and useful.

\section{Aims and objectives}

High-quality studies regarding attitudes towards e-Health are severely lacking [9]. Throughout the healthcare sector conflict between utilization of technology in EHR and preserving patient's privacy regarding their medical history remains as a major issue $[10,11]$. Physicians are faced with many issues while using the EHR, which leads to troubled outcomes [12].

This paper aims to investigate expectations, issues, and challenges in the area of EHR. The main objective of this study is to identify the EHR issues, challenges related to physicians and consumers that are significant in the development of EHR. A brief overview of laws, standards and developments of EHR along with recommendations and future directions are explored.

\section{Background}

The EHR information has several implications in the decisionmaking process in patient care and health policies [10]. As patient records are digitized, the fundamental right that is most challenged is patient-privacy [6]. Another significant barrier to the widespread use of EHR is the reluctance on the part of physicians related to EHR usage [13].

*Corresponding author: Neethu Mathai, Department of Information Technology, Murdoch University, Perth, Australia, Tel: 61401401520; E-mail: neethu856@gmail.com

Received April 05, 2017; Accepted June 19, 2017; Published June 26, 2017

Citation: Mathai N, Shiratudin MF, Sohel F (2017) Electronic Health Record Management: Expectations, Issues, and Challenges. J Health Med Informat 8: 265. doi: 10.4172/2157-7420.1000265

Copyright: $\odot 2017$ Mathai N, et al. This is an open-access article distributed under the terms of the Creative Commons Attribution License, which permits unrestricted use, distribution, and reproduction in any medium, provided the original author and source are credited. 
Consumers are in a constant fear of misuse of their health details even by the authorized personnel [12]. They would expect to establish proper privacy and security legislations to define patient's rights for disclosure of health information. Surveys indicate that the public is particularly anxious about privacy regarding health-related issues. With accidental damage, intentional misuse, and even malware, hospitals report data loss and corrupted incidents [13]. As more hospitals go digital with medical records, the frequency of data breaches are rising which shows inadequate data security that can affect huge numbers of people $[13,14]$.

The use of EHR software has significant impacts on the productivity of the health providers. Healthcare providers have certain legal and ethical obligations for securely exchanging the health information. Unfortunately, physicians are provided with minimal training for achieving this goal $[15,16]$.

The previous literature suggests that a relevant high quality study which examines the issues and expectations of consumers and physicians in EHR implementation were missing. This paper aims to investigate expectations, issues, and challenge concerning Electronic Health Records.

\section{Methodology}

The methodology of this study is through review of past literature in journals, conference proceedings and articles of expert authors. For data collection, health websites and public repositories are examined. All the databases including IEEE, Springer, ACM, and Science Direct are explored and research papers within past five years are studied [17]. The results of the studies are then analysed and solutions recommended.

\section{Findings}

The findings regarding the issues and expectations of the consumers and physicians are described in this section.

\section{Consumer issues}

The advent of electronic health records increased the convenience and feasibility of healthcare administration. However, consumers are in constant fear about the integrity and privacy of their health information [18].

\section{Privacy issues}

Security and privacy are primary concerns with strong legal and ethical implications. Privacy breaches in health sector along with complex and conflicting health privacy laws have resulted in a decrease in patients trust that their health information privacy will be protected. If privacy rights for sensitive health information are weaker than the privacy rights of individuals for less sensitive non-health data then public trust in the health care delivery system cannot be maintained [19].

\section{Data breaches}

It is reported that authorized providers use their smartphones that house potentially sensitive information for sending and receiving personal emails, browsing the web and viewing media. Reports show that $80 \%$ of health care personnel use their smartphones for workrelated tasks $[20,21]$. The system thus becomes an environment, where a whole ecology of applications may be coexisting in the device opening the door to many unintentional but malicious threats [22]. The affected individuals by the loss of electronic media were greater than those affected by unauthorized accesses or human error.
Internal employees sometimes share their password and send emails to the wrong addressee and consequently disclose the personal details of some patients. Sometimes they do not follow formal procedures and are unintentionally given the wrong privileges or authorizations. Moreover, unauthorized access consists of credit card hacking of patients and system or unauthorized employees accessing protected health information [23]. These incidents of data breaches incur an impact on reducing the trust of EHR system in individuals.

Technology applied to data security can reduce these risks but cannot avoid them entirely. Nevertheless, the protection of EHR security is a key element for any successful implementation of EHRs because it is a condition of their social reliability and acceptability [24].

\section{Medical identity theft}

The advancement of health care technologies created more opportunities for culprits to commit identity theft. EHRs allow for a larger segment of the population to be exposed to medical identity theft. Medical identity theft is the illegal access by using a patient's personal information to obtain medical services or goods. Also, the data can be sold to larger organized crime groups throughout the world [25].

Once a medical record has been altered by a criminal, it could be potentially harmful to the victim if they receive improper treatment based on the corrupt information within their file. More patient data resides in the computer systems, as more healthcare organizations move to an electronic medical record format. The ability to download thousands of records from a computer $[26,27]$ terminal many miles away makes the theft of medical records very attractive. As medical facilities have new patients every day, there are always new identities to steal and make systems more attractive for repeated attacks. Once a medical identity theft has occurred, getting a medical record amended can be a very challenging job because of the threatening changes involved $[28,29]$.

\section{Consumer expectation}

Population-based surveys suggest that patients desire online access to their medical records and email communication with clinicians [21]. Patients expect the capability of controlling accesses to their data and hiding some of their data from health care practitioners. The administration process of security settings should be easy to handle for patients to ensure about healthcare benefits [23]. There is a need of establishing well formulated and well-integrated security, privacy and confidentiality policies in the health industry.

According to research that carried out by the University of California at Berkeley, the second important concern about patient's privacy is conflicting regulatory frameworks (conflicting of federal and state regulatory frameworks with regards to the health policies) [23]. It should provide improved legibility of patient data, simultaneous remote access, and integration with other information sources [25].

In a survey of British citizens concerning the introduction of the health records system and inquired whether there were items and details which they preferred not to be shared with the national records system. They found that the items participants tended to wish to keep to them were mostly related to pregnancy, contraception, sexual health, and mental health [30]. A more recent study [31] showed that simply getting people to disclose medical information that would be digitally stored depends on a complex array of factors and circumstances, including their emotional response to several medical conditions and several contextual factors [30]. 
Previous research on patient views has identified three important factors that influence individual's decisions to allow their health information to be used: (1) The perceived sensitivity of the data; (2) The nature of patient interaction and trust in the data recipient; and (3) The extent to which individuals feel informed about how their data will be used [4].

In a survey in U.K. of 5331 individuals, most members of the public expect to be asked for explicit consent before their health data stored within integrated EHRs is shared for health provision, research, and policy [4].

\section{Physician issues}

The development of Electronic health records has a lot of positive as well as negative impacts on the physicians. Some of the issues faced by the physicians are described in this section.

Low productivity: Most EHR user interfaces are not intuitive. They do not follow physician's normal workflows. In a report on EHRs, the Institute of Medicine pointed to the poor interface design that takes away the clinician efficiency for the system, and noted that these problems can lead to underuse or misuse of the system [24]. That is, the system should support them, not work against them. Many EHR screens will show an enormous amount of headers which disrupts normal workflow [24]. Cluttered, dense screen design with lots of tabs and hyperlinks to other system screens is also disrupting the workflow. All the physicians think they lost too much time in data entry $[26,27]$. There was a sense from the physicians that there was a net negative effect of the technology on their work practices and productivity.

The surveyed data indicate that physicians are encountering difficulties when communicating and collaborating with RNs (registered nurses) and specialty areas. The biggest area of concern is the lack of concurrent access which results in productivity loss, communication inefficiency, and collaboration interference [16].

Overwhelmed alerts: Findings from a new study show that some physicians receive more than 100 notifications per day via Electronic Health Record (EHR)-based inboxes and dealing with this electronic burden requires more than an hour every day. Excessive EHR-based notifications can overburden physicians, and lead to potentially disastrous consequences. Unmanageable numbers of alerts not only make it difficult for physicians to filter out important information but also increase the chances that physicians will miss patient's test results [28].

Reluctance in using HER: Highly demanding educational and specialized training are given to physicians. Prior research suggests that physicians are reluctant in giving a positive response to Information System implementation that interferes with their existing routines [16]. Research highlights that the physician's response to the lack of support for their knowledge needs is to request additional system enhancements for their work and data access.

When it comes to health information technology, negative patient attitudes toward EHRs may contribute to decisions by their health care providers to reject EHR use in clinical practice [27]. Additionally, physicians cannot share patient health information electronically with other health care providers without obtaining patient consent [27].

\section{Physician expectations}

Patient care process includes the process of getting data, turning it into information and then to use it in the decision-making. If physicians do not have the enough information about the medical history of the patient they would not be able to give the right decisions. To be able to offer a better healthcare diagnostics and treatments, health care providers should have good information about the patient's situation. Keeping accurate and correct information is important otherwise with wrong data, wrong clinician actions can be taken on the patients and data access improvements $[19,22]$.

The physicians indicate concerns with slow response time. These concerns lessen the EHR availability, and the access of the system affects productivity as well as patient care. Also, there is a concern with the hardware and configuration area. The research data indicate physicians want to utilize various hardware devices, such as tablet PCs for their work. They voice dissatisfaction with the present EHR interface. Hence, the frustration among the physicians and their loss of productivity because of EHR persists [16].

In a survey of physicians in the province of Québec City in Canada, $64 \%$ of them think it is better than on accessing an EHR, physicians should be allowed to view the entire content of the patient's EHR thereby excluding the possibility of masking certain information [4].

Contrary to other studies, sociodemographic characteristics did not significantly influence physician intention to use EHR [4]. Social Norm (SN) is also significantly associated with EHR adoption by a physician. Physician's decision regarding EHR adoption could also be influenced by their peers [4].

\section{Physician challenges}

Physician's acceptance of EHR systems is a critical factor for a successful implementation. Physicians resist the technology due to productivity issues, workflow challenges, lack of support and other issues. This leads to high costs and reduction in unable to move easily between screens [32]. Physicians feel that EHR interferes with the way they organize their thought processes on patient care. Understanding how physicians work with knowledge in the healthcare domain is an important step in understanding the physician's perspective on EHR usage [33]. The challenges in EHR adoption by physicians have tempered efforts to improve the efficiency of healthcare through the technology. Providing access to medical information between different providers enables health care professionals from different organizations to function as a unit, and helps to prevent double testing which can cut costs $[34,35]$.

\section{Various laws, standards and developments in EHR}

The emergence of electronic health records (EHRs) causes organizations to define and disclose information. Information in EHRs is often stored in multiple systems. To achieve higher quality care legal, ethical, and financial issues need to be addressed. Further studies and conversations involving patients, lawyers, ethicists, economists, policymakers, technology experts and clinicians have to be done.

For years healthcare organizations have struggled to align legal health records with designated record set required by the HIPAA privacy rule. The healthcare organization must explicitly define both in a multidisciplinary team approach.

The HITECH Act established provides the U.S. Department of Health and Human Services with the authority to establish programs to improve health care quality, safety, and efficiency. Improvement is achieved through the promotion of health IT, including EHRs private and secure electronic health information exchange. 
Citation: Mathai N, Shiratudin MF, Sohel F (2017) Electronic Health Record Management: Expectations, Issues, and Challenges. J Health Med Informat 8: 265. doi: 10.4172/2157-7420.1000265

Canada Health Info way with 1.1 billion dollar budget is a major investment for Canadians which would support more efficient healthcare delivery, and patient confidentiality while enabling better decisions about diagnosis and treatment.

In England, National Program for Information Technology, directed by the National Health Service (NHS) is responsible for delivering EHR. The system uses a unique identifier for each patient which would be able to have a summary of their important health information, known as their Summary Care Record (SCR). SCR is accessed using a secure website named as "Health Space".

In Australia, Health Connect is a joint Australian, State and Territory Governments initiative for revolving paper-based health records to EHRs for the benefit of consumers and also health-care providers. Health Information Technology (HIT) is considered the basis for improving the quality of healthcare, safety and efficiency by the government.

EHR development and standards are being developed in Indonesia, Korea, Malaysia and several other developing countries and mainly concerned with the hospital-based environment.

\section{Recommendations}

The confidentiality, privacy problem regarding consumers can be addressed by the implementation of strong cyber health laws in each country after conducting a broad survey of the consumers. In the survey, consumers can express their expectations and issues they are facing with EHR. Considering the majority opinion, the governments can discuss and come up with strong laws. Medical identity theft and data breaches can be prevented to a large extent by strong cyber health laws along with powerful encrypted data storage and exchange in EHR systems. Awareness classes regarding the implemented privacy laws, and EHR usage should be given to consumers.

Poor EHR design is hindering the workflow of physicians. EHR systems should be redesigned to support the workflow of clinicians. For managing chronic illness, increased interoperability between EHR systems and patient's mobile technologies and telehealth technologies need to be developed. Moreover proper training regarding EHR, its usage and privacy rights must be given to physicians.

\section{Conclusion}

Electronic Health Records (EHR) contain medical data about a specific individual in electronic format. EHR systems are the software platforms that health care service providers (e.g., hospitals, clinics) use to maintain EHR information of patients. The main focal point for literature review consisted of online database and articles. This research paper focused on expectations, issues and challenges faced by the consumers and physicians in the implementation of EHR. The work identified the main issues concerning consumers as data breaches, privacy issues and medical identity theft. Low productivity, overwhelmed alerts and EHR reluctance are the major physician issues. Solving the issues will pave the way for a remarkable contribution in EHR development process.

\section{Future Work}

Further public discussion about Electronic Health Records is needed to develop an efficient system of proposed data sharing arrangements between patients and health providers. Moreover, quantitative studies including surveys covering a large number of consumers as well as physicians have to be conducted for better results. Questionnaires and interviews can be carried out to get more public expectations about Electronic Health Records.

\section{References}

1. Handel DA, Hackman JL (2010) "Implementing electronic health records in the emergency department.” J Emerg Med 38: 257-263.

2. Acharya S, Coats B, Saluja A, Fuller D (2013) "Secure Electronic Health Record Exchange: Achieving the Meaningful Use Objectives." Int Conf Sys Sci 46: 1-10.

3. Rosenthal DI (2013) “Instant Replay.” Healthcare 1: 52-54.

4. Nocco L, Peigné V (2014) "An empirical study of healthcare providers and patient's perceptions of electronic health records." Compu Bio Med 59: 194-201.

5. Caligtan CA (2011) "Electronic health records and personal health recordsseminars in oncology nursing." Semin Onco Nurs 27: 218-228.

6. Buckley BS, Murphy AW, MacFarlane AE (2011) "Public attitudes to the use in research of personal health information from general practitioner's records: a survey of the Irish general public." J Med Ethic 37: 50-55.

7. Kumar P, Sridhar KV, Prasad KSR (2011) "A new paradigm of electronic health record for efficient implementation of health care delivery." CSDL 1: 118-120.

8. Tang PC, Ash JS, Bates DW (2006) "Personal health records: definitions, benefits, and strategies for overcoming barriers to adoption." J Ameri Med Informat Ass 13: 121-126.

9. Chen C, Garrido T, Chock D, Okawa G, Liang L (2009) "The Kaiser Permanente electronic health record: transforming and streamlining modalities of care." Healt Aff 28: 323-333.

10. Institute of Medicine (2001) "Crossing the quality chasm: a new health system for the $21^{\text {st }}$ century." The National Academies Press, DC, USA.

11. Hayrinen K (2008) "Definition, structure, content, use and impacts of electronic health records: a review of the research literature." Int J Med Informat 77: 291-304

12. Smith M (2015) "Electronic health records and healthcare identifiers: legislation discussion paper." Population Health and Research Network.

13. Garrety K, McLoughlin I, Wilson R, Zelle G, Martin M (2014) "National electronic health records and the digital disruption of moral orders." Soc Sci Med 101: 70-77.

14. Kwon I, Johnson ME (2015) "Protecting patient data-the economic perspective of healthcare security." IEEE 13: 90-95.

15. Burns AJ, Johnson ME (2015) “Securing health information." IT Profes 17: 23-29.

16. Noteboom CB, Motorny SP, Qureshi S, Sarnikar S (2014) "Meaningful use of electronic health records for physician collaboration: A patient centred health care perspective." IEEE 1: 656-666.

17. Current Consumer Research (2010) "eHealth and electronic health records: consumer perspectives and consumer engagement."

18. Xanthidis D, Aleisa E (2012) "eHealth record and personal privacy." Int Conf Informat Tech e-Servic 1: 1-8.

19. Kok OM, Başoglu N (2012) "Exploring the success factors of electronic health record systems adoption.” IEEE 1: 1471-1477.

20. Terasa P, Nadi S (2011) "Selection of Electronic health records." IEEE 1: 1- 6.

21. Walker J, Leveille SG, Ngo L, Vodicka E, Darer JD, et al. (2012) "Inviting patients to read their doctor's notes: patients and doctors look ahead." Anna Int Med 155: 811-819.

22. Markle Foundation (2011) "Markle survey: the public and doctors agree on importance of specific privacy protections for health I.T", Markle Survey on Health in a Networked Life.

23. Rezaeibagha F (2013) "Privacy and data security of electronic patient records (epr) sharing", Master of Science.

24. MEDHOST Solutions Corp (2013) "It's time to meet physicians' concerns about ehrs."

25. Portela $F(2010)$ "Electronic health records in the emergency room." IEEE 1: 195-200.

26. Grana M, Jackwoski K (2015) "Electronic health record: A review." IEEE 1 1375-1382. 
Citation: Mathai N, Shiratudin MF, Sohel F (2017) Electronic Health Record Management: Expectations, Issues, and Challenges. J Health Med Informat 8: 265. doi: 10.4172/2157-7420.1000265

Page 5 of 5

27. Pereira R, Duarte J, Salazar M, Santos M, Abelha A, et al. (2012) "Usability of an electronic health record." IEEE 1: 1568-1572.

28. Steininger K, Stiglbauer B, Baumgartner B, Engleder B (2014) "Factors explaining physician's acceptance of electronic health records." IEEE 1: 27682777.

29. Ben-Assuli O (2014) "Electronic health records, adoption, quality of care, legal and privacy issues and their implementation in emergency departments." Health Policy 119: 287-297.

30. Anderson CL, Agarwal R (2011) "The digitization of healthcare: bound-ary risks, emotion, and consumer willingness to disclosepersonal health information." Informat Sys Res 22: 469-490.

31. Jensen L, Bossen C (2016) “Factors affecting physician's use of a dedicated overview interface in an electronic health record: The importance of standard information and standard documentation." Int J Med Informat 87: 44-53.

32. Quia Y, Asan O, Montague E (2015) "Factors associated with patient trust in electronic health records used in a primary care setting: Results from a survey." Health Policy and Technology 4: 357-363.

33. Irvine C, Therasa $F$ (2009) "Consumer privacy issues associated with the use of electronic health records." Acad Healt Manag J 5: 2.

34. Patterson (2014) "Fifth annual study on medical identity theft". News Publications.

35. Thurston J (2014) "Meaningful use of electronic health records-the journal for nurse practitioners." J Nurs Pract 10: 510-513. 